\title{
LAS RAÍCES FILOSÓFICAS Y FÍSICAS DE LAS HIPÓTESIS SOBRE SISMOLOGÍA DESARROLLADAS POR EL ING. LUIS MATAMOROS. UNA INFLUENCIA DE LAS CORRIENTES ARISTOTÉLICAS Y FEIJONIANAS A INICIOS DEL SIGLO XX
}

\author{
Giovanni Peraldo Huertas ${ }^{1 *} \&$ Jorge A. Amador ${ }^{2}$ \\ ${ }^{1}$ Escuela Centroamericana de Geología, Universidad de Costa Rica. \\ Apdo. 214-2060, Ciudad Universitaria Rodrigo Facio \\ ${ }^{2}$ Centro de Investigaciones Geofísicas (CIGEFI), Universidad de Costa Rica \\ *Autor para contacto: gperaldo@geologia.ucr.ac.cr
}

(Recibido: 10/06/07; aceptado: 20/12/07)

\begin{abstract}
This paper deals with the research carried out by Eng. Luis Matamoros Sandoval, who in 1902 published in Costa Rica his investigations on seismology in an article entitled "El interior de la corteza terrestre: una nueva teoría sobre el origen de los temblores" (The interior of the Earth's crust: a new theory about the origin of earthquakes). In his work, Matamoros followed the Aristotelic current and mixed it with the hypothesis developed by the Spanish friar Benito Feijoo, who proposed electricity as the physical cause of intense earthquakes perceived over large regions. Matamoros justifies his results by the use of mathematics combined with fundamental physical principles, some which he had great difficulty to explain due in part to the use of criteria and basic concepts that were not supported by the traditional physical knowledge. Despite his conceptual errors, the value of his work resides in the fact that it was the first local formal scientific attempt to explain the origin of earthquakes, a process that even in the great scientific centers of the period was the source of continuous speculation.
\end{abstract}

Key words: History, Seismology, $20^{\text {th }}$ century, Luis Matamoros, Costa Rica.

RESUMEN: Este trabajo trata sobre la investigación realizada por el Ing. Luis Matamoros Sandoval, quien en 1902 publicó en Costa Rica sus investigaciones en Sismología en un artículo denominado "El interior de la corteza terrestre: una nueva teoría sobre el origen de los temblores". En su trabajo, Matamoros sigue la corriente de pensamiento aristotélica y la mezcla con las hipótesis del fraile español Benito Feijoo, quien propone la electricidad como la causante física de intensos terremotos con una gran área de percepción. Matamoros justifica sus resultados con matemática combinada con principios fundamentales de la física, los cuales explica en parte con cierto grado de dificultad, pues utiliza criterios y conceptos que no estaban soportados por el conocimiento de la física tradicional. Pese a los errores conceptuales, el trabajo es valioso por ser el primer intento científico local formal por explicar el origen de los temblores, proceso que aún en los grandes centros científicos de la época era fuente de continua especulación.

Palabras clave: Historia, Sismología, siglo XX, Luis Matamoros, Costa Rica. 


\section{INTRODUCCIÓN}

Dentro del patrimonio científico de Costa Rica que trata de temas sismológicos, destaca el libro "Dinámica interna del Globo", publicado en 1902 por el Ing. Luis Matamoros Sandoval, e impreso en la Tipografía Nacional (Fig. 1). En ese libro, Matamoros propone una hipótesis que mezcla dos corrientes filosóficas sobre el origen de los sismos y apoya sus ideas mediante un planteamiento físico-matemático para demostrar sus hipótesis con relación a la generación de sismos.

En este artículo se discuten las hipótesis planteadas por Matamoros a la luz del conocimiento actual geológico, sismológico y físico. $\mathrm{Si}$ bien es cierto que en la actualidad las hipótesis planteadas por Matamoros han sido superadas por las nuevas investigaciones geofísicas, para el tiempo en que se escribieron eran hipótesis aceptadas en círculos académicos de Costa Rica y del mundo, pues si algunos las rechazaban como falsas, otros mantenían su apoyo a la corriente del pensamiento en que se adecuaban. Este aparente caos de ideas sobre el origen de los sismos se entiende porque en esos años la Sismología, como ciencia, apenas estaba surgiendo y se estaban construyendo sus bases cognitivas recurriendo en múltiples oportunidades a los conocimientos de la antigüedad clásica o a hipótesis formuladas durante la época de la Ilustración. Además, la Geología no llegaba aún a los planteamientos de teorías como la Tectónica de Placas, que dio la base de la explicación geológica actual sobre el origen de los sismos.

La investigación desarrollada por el Ing. Matamoros fue motivada, en parte, por las observaciones que él realizó de los daños ocasionados por el terremoto de Fraijanes del 30 de diciembre de 1888. En el círculo científico nacional, el documento fue acogido con entusiasmo por tres razones: 1) esos círculos científicos académicos costarricenses eran integrados por personas que tenían conocimientos empíricos en temas geológicos, pues casi ninguno de los pensadores del momento tuvieron especialidad en Geología; 2) los científicos con especialidad en esa ciencia aún seguían ideas relacionadas con el pensamiento aristotélico adaptado hacia

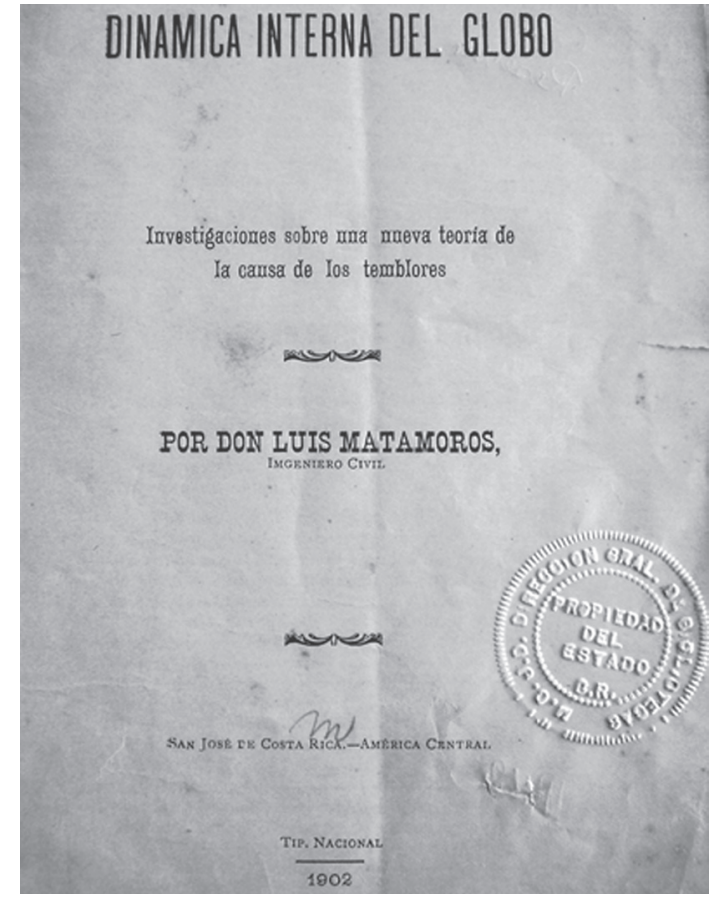

Fig. 1: Portada del libro del Ing. Luis Matamoros Sandoval que se localiza en la Biblioteca Nacional de Costa Rica.

la tendencia del pensamiento científico de la época que apoyaba la idea, entre otras, de que los volcanes eran las causas de los sismos, y 3 ) la Sismología estaba aún en sus primeros pasos y la causa de los sismos era un tema que quedaba casi a la elucubración. Se podría comparar el "corpus" cognitivo en Sismología con el actual debate sobre cambio climático.

En este sentido, muchos de los que lean a Matamoros, pueden creer estar frente a un gran conjunto de sofismas. Sin embargo, si se analiza a fondo el trabajo de Matamoros, este se esforzó en demostrar una corriente de hipótesis aceptadas en su tiempo, o al menos no rechazadas de plano. Se esforzó por dar cohesión a su trabajo entre las ideas prevalecientes y las demostraciones matemáticas para que resultase una hipótesis coherente. De hecho, las ideas se apoyan en el conocimiento del momento derivado de la evolución del pensamiento, fortalecido por pensadores clave que influenciaron en su tiempo la reflexión científica, más si estos pensadores fueron reconocidos por sus iguales. Tal como fue el caso del benedictino 
Benito Feijoo (1676 - 1764), quien esbozó su hipótesis sobre la electricidad como causa de los sismos pues en esa época los científicos “... recurren a ella [la electricidad] para explicar la causa de los truenos, y rayos..." (Feijoo, 1760a).

\section{LA VIDA Y OBRA DEL ING. LUIS MATAMOROS}

Don Luis Matamoros Sandoval (Fig. 2) nació en el año 1859 y murió en 1934 (Díaz, 2005). En su vida profesional se desempeñó en diversas actividades del quehacer nacional. En el Instituto Nacional impartió lecciones de Cálculo Infinitesimal, Topografía y Mecánica Racional a finales de la década de 1870 (Ruiz, 2003).

Faerrón (en La Tribuna del 24 de enero de 1934) refiere que la labor más grande que el Ing. Matamoros realizó para el país fue su participación como “....consejero técnico de la Delegación constituida cerca del Chief Justice de los Estados Unidos de Norteamérica, encargado por los gobiernos de Costa Rica y Panamá de declarar la recta interpretación del Laudo Lambert...".

El 18 de julio de 1903 se le eligió presidente de la Facultad Técnica de la República (www. cfia.or.cr/resenna.htm). Esta organización se fundó el 3 de julio de 1903 mediante decreto por el presidente Ascensión Esquivel Ibarra y dio origen al actual Colegio Federado de Ingenieros y Arquitectos de Costa Rica.

Don Luis Matamoros fue un ingeniero que laboró en los planes y fiscalización de muchas obras de importancia de finales del siglo XIX y principios del XX, tal como se observa en el Cuadro 1. Fue funcionario del Ministerio de Fomento (actual Ministerio de Obras Públicas) y como ingeniero de esta dependencia realizó una valiosa contribución al cantón de Puriscal, cuando en 1914 estudió las nacientes existentes en la ciudad de Santiago, que ya para esa época tenía problemas de abastecimiento de agua potable (Peraldo, 1996).

También incursionó en la minería, al traducir en 1886 en el periódico La Gaceta, un método para el beneficiado de metales que incorporaba la electricidad para estimular el proceso de la hidrólisis. A este método le aportó sugerencias para mejorar la eficiencia de la máquina (Peraldo, 2003).

Escribió muchos artículos que están referenciados en la Biblioteca Nacional, que se muestran en el Cuadro 2. Se observa que incursionó en diversos temas que van desde la geografía política, reglamentos para el sector ingenieril de la Secretaría de Fomento, hasta el estudio del recurso hídrico.

\section{IDEAS SOBRE LA GENERACIÓN DE SISMOS}

Para entender el pensamiento que Luis Matamoros defiende en su obra "Dinámica interna del globo", es imprescindible abarcar un poco de la historia de la evolución del pensamiento sobre las hipótesis planteadas en diferentes épocas sobre el origen de los sismos.

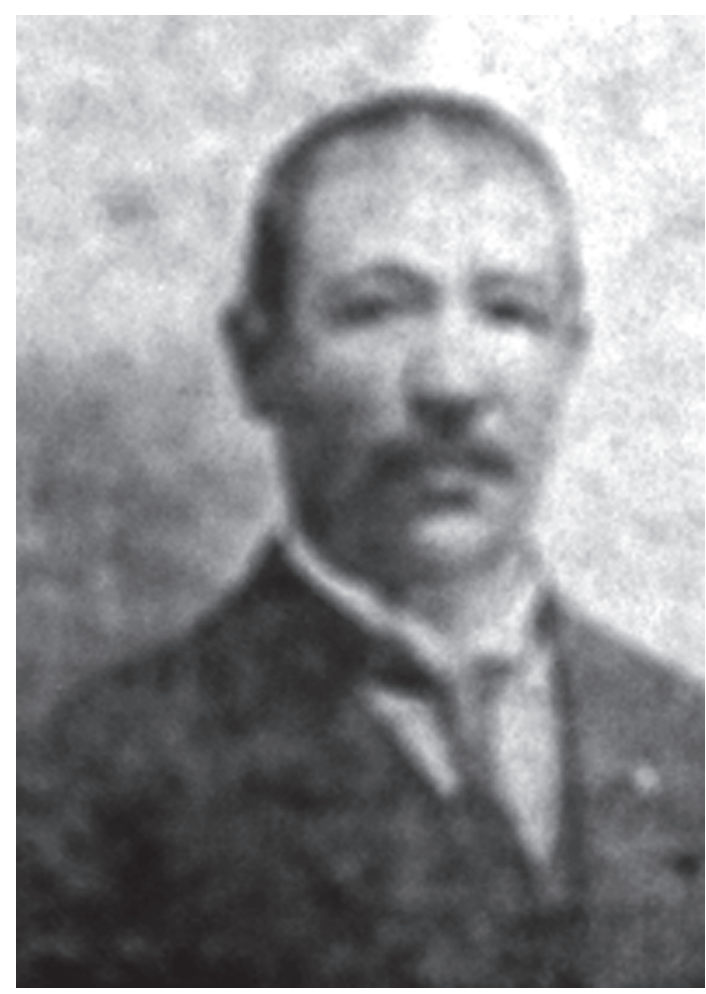

Fig. 2: Fotografía del Ing. Luis Matamoros Sandoval (Tomada de La Tribuna, 1913?). 
Cuadro 1

Obras de infraestructura aprobadas o creadas por don Luis Matamoros

\begin{tabular}{cccc}
\hline Año & Obra en planos & Lugar & Fuente \\
\hline 1877 & Edificio de dos plantas & Desconocido & ANCR, Planos 15927 \\
1890 & Mapa de límites Nicaragua-Costa Rica & entre Salinas y Sapoa & ANCR, Planos 20860 \\
1894 & Cuartel & Alajuela & ANCR, Planos 1244 \\
1895 & Diseño portada panteón & San Joaquín, Heredia & ANCR, Planos 12515 \\
1901 & Desvío de aguas, acueducto & Para Liberia & ANCR, Planos 839 \\
1906 & Codiseño, cañería & Juan Viñas & ANCR, Planos 21616 \\
1906 & Diseño cañería & Alajuelita y Hatillo & ANCR, Planos 12961 \\
1907 & Cárcel & Tucurrique & ANCR, Planos 203 \\
1907 & Codiseño cañería & San Sebastián & ANCR, Planos 12968 \\
1907 & Plano escuela & San José, Alajuela & ANCR, Planos 20845 \\
1912 & Planta hidroeléctrica & Limón & ANCR, Planos 12511 \\
1912 & Codiseño cañería & Cañas, Guanacaste & ANCR, Planos 19988 \\
\hline
\end{tabular}

En épocas pasadas como en las actuales el origen de los sismos es interpretado de diferente manera, debido a la existencia de dos grupos de pensamiento que pueden agruparse en formales e informales. García (2001) indica que la leyenda y el mito, la tradición oral y el conocimiento popular se conjugan para buscar las causas que originan los sismos. Generalmente ese pensamiento popular presenta elementos del pensamiento formal y del informal.

Los griegos de la antigüedad clásica desarrollaron ideas para explicar a su pueblo el origen de los sismos. García (2001) menciona las ideas de Aristóteles que lograron llegar hasta nuestros días. Esta hipótesis aristotélica refiere que en el centro de la tierra existe un fuego permanente que genera gases o exhalaciones que al desplazarse en el interior de la tierra provocan los temblores. Otros filósofos como Lucrecio y Séneca contribuyeron a las hipótesis sobre el origen de los sismos (García, 2001), aunque estos dos pensadores claramente tienen una marcada influencia aristotélica.

En el siglo XIX la corriente de pensamiento aristotélica - senequista - lucreciana fue modificada y difundida por científicos tales como Humboldt. Este planteaba que los grandes sismos que no son acompañados por erupciones volcánicas tienen su origen en los vientos que se escapan violentamente de la tierra (Peraldo, 2003).

Otras hipótesis tratan de asociar la sismicidad con corrientes eléctricas y datan del siglo XVIII y su autor fue Benito Feijoo (García, 2001). Benito Feijoo fue un filósofo español representante de la Ilustración, quien nació en 1676 y murió en 1764. Sus ideas sobre el origen de los sismos, las desarrolla motivado por el sismo que destruyó Lisboa en 1755 (Feijoo, 1760b).

Feijoo en la carta XXVIII de "Cartas eruditas y curiosas" tomo $\mathrm{V}$, propone que los sismos ocurren por dos causas que son: 1) desplome de bóvedas en cavernas y 2) incineración de materias sulfúreas, bituminosas, nitrosas, entre otras. Pero refiere que estas explicaciones calzan perfectamente para explicar sismos muy locales, pero para explicar por medio de las anteriores el sismo de Lisboa, que al mismo tiempo que temblaba en esa ciudad portuguesa, ocurría lo mismo en la Andalucía de España, la explicación debe ser otra pues le parece inexplicable que en todos los pueblos donde se sintieron sismos ese día y casi a la misma hora, existieran desplomes de bóvedas en cavernas o que existiera la coincidencia de la incineración de las materias que antes se anotaron. Entonces, aludiendo a experimentos con electricidad, efectuados en algunas ciudades europeas, 
Cuadro 2

Publicaciones de Luis Matamoros asequibles en la Biblioteca Nacional de Costa Rica.

\begin{tabular}{|c|c|}
\hline Publicación & Signatura \\
\hline 1884: A propósito de un estudio sobre el ferrocarril del Pacífico. La Enseñanza, 1 (7), agosto. & $\mathrm{H} 370.5$ / E59e CR \\
\hline 1884: Noticias sobre el puente del Barranca. La Enseñanza, 1 (8). & H370.5 / E59e CR \\
\hline $\begin{array}{l}\text { 1894: Circular N } 1 \text { a los señores ingenieros y ayudantes de la Dirección e Inspección General de } \\
\text { Obras Públicas. San José, Costa Rica, Tipografía Nacional, } 11 \text { págs. }\end{array}$ & $625.76 \mathrm{M}$ \\
\hline $\begin{array}{l}\text { Con Leonidas Carranza, 1897: Exposition submitted by the Costa Rican boundary Comissión } \\
\text { To Hon. E.P. Alexander Engineer Artibration. San José, Tipografía Nacional }\end{array}$ & 917.286 / M425 - e \\
\hline $\begin{array}{l}\text { 1902: Dinámica interna del globo; investigaciones sobre una nueva teoría de la causa de los } \\
\text { temblores. San José, Tipografía Nacional, } 26 \text { págs. }\end{array}$ & $551.2 / \mathrm{M} 425 \mathrm{~d}$ \\
\hline $\begin{array}{l}\text { 1902: Documentación del proyecto de cañería de San José. San José, Costa Rica, Tipografía } \\
\text { Nacional, } 121 \text { págs + planos. }\end{array}$ & $352 \mathrm{M}$ \\
\hline $\begin{array}{l}\text { 1906: Circular N } 49 \text { y reglamento y principios generales que deben atenderse en la composición } \\
\text { de los caminos y carreteras nacionales. San José, Costa Rica, Tipografía Nacional, } 12 \text { págs. }\end{array}$ & $625.76 \mathrm{M}$ \\
\hline 1910: El valor de las fuerzas hidráulicas en Costa Rica. Páginas Ilustradas, año 7, enero 1910. & H056P \\
\hline $\begin{array}{l}\text { 1913: Costa Rica - Panamá arbitration report submitted to the representative of Costa Rica. } \\
\text { Washington, Press of Gibson. }\end{array}$ & CR917.286 / M425c \\
\hline $\begin{array}{l}\text { 1913: Costa Rica - Panamá arbitration, Washington, Evidence for determining the position of } \\
\text { the boundary line between the republics of Costa Rica and Panamá. }\end{array}$ & CR $917.286 \mathrm{M} 425 \mathrm{c}$ \\
\hline $\begin{array}{l}\text { 1915: Informe del ingeniero don Luis Matamoros resolviendo las cuestiones propuestas por el } \\
\text { regidor Lic. D. Joaquín Aguilar aprobadas por la Corporación Municipal de la Ciudad de San } \\
\text { José. San José, Tipografía de San José, } 25 \text { págs. }\end{array}$ & CR628.1 / M425i \\
\hline $\begin{array}{c}\text { Con Luciano Fernández, 1915: Refutaciones hechas por la Junta de Saneamiento al informe } \\
\text { del ingeniero municipal y contestación del ingeniero municipal don Luis Matamoros. San José, } \\
\text { Costa Rica, Imprenta Alsina (fotos, tablas, nota: a la cabeza del título: Cañería y cloacas de la } \\
\text { ciudad de San José.) }\end{array}$ & $628.15 \mathrm{~F}$ \\
\hline
\end{tabular}

se vio que casi al mismo tiempo que se aplica electricidad a un extremo de una cadena humana, esta es sentida por la última persona que forma la cadena, e invoca los experimentos de Leyden que fueron replicados en Versalles por el Abat Nollet (Feijoo, 1998a). El experimento de Leyden es de vital importancia para la tecnología que usa electricidad porque es el precursor del acumulador (Wildermann, 1911). Esta idea sirvió para imaginar acumuladores naturales en la tierra.

Lo anterior le permitió a Feijoo, interpretar que la materia eléctrica fue la responsable de afectar cúmulos de materias incandescentes en un ámbito corto de tiempo. En su alocución, Feijoo resalta que estas materias incandescentes son cúmulos eléctricos que pueden dispararse por algo que llama golpe fulminante. Si bien Feijoo se basa en hipótesis actualmente incompatibles con la
Sismología, sus esfuerzos por explicar los sismos produjeron enunciados no tan erróneos a la vista de los conocimientos actuales. Comentarios muy interesantes ponen a Feijoo en una posición de precursor de la Sismología actual, tal como el siguiente: “...cuanto más terribles, y comprehensivos de mayor espacio son los Terremotos, tanto menos temibles sus repeticiones..." La razón física que expone es que al ser, como el de Lisboa de 1755 tan grande más materia eléctrica consumió y por lo tanto que “....por vía de nueva producción o por afluencia de la contenida en partes distantes, se reponga igual cantidad de materia..." (Feijoo, 1760c).

Darwin por su parte pensaba que los temblores, como el que presenció en 1835 en Concepción, Chile eran el producto del desgarramiento de las capas rocosas cuando se infiltran rocas en estado 
líquido (Peraldo, 2003). Una idea cercana a las que existen en la actualidad, para asociar sismos a la actividad volcánica.

Costa Rica, no tuvo durante el siglo XIX la importancia de otros países americanos en cuanto a ser centro de actividad científica como fue México. Sin embargo, nuestra diminuta actividad académica y científica, concentrada en la otrora Universidad de Santo Tomás, produjo personas con inquietudes intelectuales con alta preocupación científica. Algunos de ellos se conocen gracias a un trabajo de investigación silente que no obstante, fue una base cognitiva a científicos europeos que llegaron al país bajo la reforma de Mauro Fernández. Un medio difusor del conocimiento para el debate fueron los periódicos circulantes en el siglo XIX. En este sentido, fue publicado en el periódico El Costarricense, en 1848 un artículo sobre la estructura del planeta (Peraldo, 2003). Dicho artículo es uno de los primeros que se conocen en los periódicos del país sobre esta temática y en donde se indicó que el planeta no es homogéneo, que fue primitivamente fluido, que su densidad aumenta hacia su centro y que las causas que alteran su superficie no influyen a profundidad. Este artículo debió dar bases para que nuestros primeros pensadores costarricenses sobre el tema de los misterios del planeta empezaran a elucubrar hipótesis.

Después, en 1852, se reprodujo en el diario La Gaceta un interesante artículo sobre postulados relacionados con el origen de los sismos. En este artículo se indicaba que sismos que abarcan un área pequeña son producidos por la dilatación de gases encerrados en las concavidades de la Tierra (Peraldo, 2003). Obsérvese que aún en la mitad del siglo XIX estaban vigentes las ideas originarias del filósofo griego Aristóteles. Rodríguez (1992) propone que la influencia del pensamiento aristotélico prevaleció hasta el siglo XVIII. No obstante, consideramos que aún en el siglo XIX y principios del XX estas ideas, modificadas, todavía prevalecían en el imaginario formal. También pueden reconocerse las ideas basadas en los planteamientos del sacerdote Feijoo.

En 1855 dos científicos alemanes radicados en Costa Rica, Francisco Kurtze y Fernando Streber, postulan el origen no eléc- trico de los sismos (González, 1910). Sin embargo, estos autores comparten la hipótesis del fuego como activador de temblores (hipótesis en la que en casi todas las explicaciones dadas, por lo menos en Costa Rica, sobre el origen de los sismos, el fuego tiene un papel principal), elemento que influenció a los investigadores españoles de los siglos XVI y XVII y que caló hondo en América (García, 2001).

Una idea que se postuló fue la relación de los sismos con aspectos astronómicos. Lardé (1960) refiere que una idea planteada es la que relaciona la sismicidad y las variaciones de las manchas solares. Cita a dos personajes científicos como el Abate Moreaux y a Kluge, quienes propusieron que las erupciones volcánicas y los sismos guardan relación con dichas variaciones y pensaban que las erupciones y temblores ocurrían cuando las manchas solares llegan a su minimun. Según Lardé (1960), esta regla permitió prever el terremoto de San Francisco y los temblores de 1908 y 1909 (que no cita donde ocurrieron), pero reconoce que ha fracasado en otros eventos, en cuyo caso señala con mucho acierto "...que han venido a probar que el problema no esta resuelto..."

Díaz et al. (2007) estudiaron el pensamiento del científico costarricense don Pedro Nolasco Gutiérrez quien fundamentó sus hipótesis de trabajo en cuanto a predicción sísmica en función de las variaciones de las manchas solares. No se conoce la opinión de Nolasco sobre las hipótesis planteadas por Matamoros. Las hipótesis de ese entonces podrían agruparse en las que proponen causas astronómicas, con lo que las hipótesis de Nolasco y algunas de Guillermo Molina no chocarían violentamente con las esgrimidas por Matamoros y las que observan las causas en aspectos relacionados con el interior del planeta, como la actividad volcánica.

El pensamiento de Henri Pittier es claro en ese particular, pues su discusión en torno al terremoto del 30 de diciembre de 1888 , lo enrumba hacia el volcán Poás como la causa de los sismos con base a los efectos superficiales que observó en su edificio volcánico. Al parecer, en Costa Rica las hipótesis sobre el origen de los sismos siguieron gravitando alrededor de los volcanes, pues el mismo Pittier aduce que el sismo del 20 de enero 
de 1905 pudo ser volcánico, aunque concluye que en el estado en que se encuentra la ciencia sismológica, no se puede asegurar nada al respecto del origen de los sismos.

Ya desde inicios del siglo XIX se empezó a analizar la relación fuerza - deformación, de manera teórica, hasta el desarrollo de una ecuación sobre la teoría de la elasticidad, por Navier y Cauchy entre 1821 y 1822 (Lay \& Wallace, 1995). En 1830 Poisson demostró, mediante desarrollos matemáticos, la existencia de dos tipos de ondas que se propagan a través de sólidos homogéneos (las ondas $\mathrm{P}$ y S) y en 1887 el sismólogo Rayleigh demuestra la existencia de otras ondas que se mueven sobre las superficies de los sólidos, que son finalmente caracterizadas en 1911, solamente que quedaron sin demostrar debido a la ausencia de tecnología capaz de aclarar su existencia (Lay \& Wallece, 1995).

Aquí se observan dos problemas de ese tiempo relacionados con la Sismología: 1) la demostración veraz de la existencia de las ondas elásticas referidas arriba, que ya de manera teórica estaban indicando la naturaleza de dicho movimiento y 2) la incertidumbre, sumamente importante sobre cuál es el origen de dichas ondas, o en otras palabras, cuál es el origen de los esfuerzos que originan en el medio sólido dichas ondas. En este sentido, la Sismología aún no solucionaba el problema de fondo: ¿qué origina la existencia de dichas ondas? De ahí que aún a principios del siglo XX siguieran sobre el tapete de discusión las ideas clásicas sobre el origen de los sismos: actividad de gases o bien, la electricidad.

\section{LA IMPORTANCIA DE SU ESCRITO "DINÁMICA INTERNA DEL GLOBO"}

Luis Matamoros tiene una gran influencia de la corriente de pensamiento de Feijoo, posiblemente reflejado en autores contemporáneos de Matamoros que postularon ideas similares en cuanto al origen eléctrico de los sismos. Trata de resolver el problema de la interpretación sobre las corrientes eléctricas, demostrando su pensamiento mediante una obra en donde el peso de la hipótesis se fundamenta en el razonamiento físico - matemático. En la última parte de su trabajo compara sus hipótesis con las que fueron publicadas en prestigiosas revistas estadounidenses y francesas y comenta como conclusión de dicha comparación que "Siendo estos documentos extremadamente novedosos, nos corresponde, pues, involucrar nuestro exclusivo derecho de propiedad intelectual del artículo preincerto".

Las hipótesis que plantea en su trabajo de 1902 , en grandes rasgos conjugan diferentes corrientes del pensamiento que han tratado de explicar la generación de los temblores. Esto reviste de importancia el escrito de Matamoros, pues somete al análisis las diversas corrientes y sintetiza un producto que refuerza con demostraciones matemáticas. Al respecto, Matamoros dedica su trabajo al geólogo francés Albert Auguste Cochón de Lapparent, quien no aceptó la hipótesis planteada, según comentó don Fidel Tristán en un opúsculo que escribió como comentario al trabajo recopilativo de Cleto González de 1910. Sin embargo, Tristán comenta que pese a lo anterior, “....esto no quiere decir que dicha hipótesis esté fuera por completo, de tantas á que tenemos que recurrir, para explicar el origen de los fenómenos sísmicos. En todo caso el estudio del Ingeniero Matamoros, tiene su mérito." Sin embargo, Matamoros resalta en su escrito cuando plantea la hipótesis del elipsoide de revolución como figura del globo terráqueo, que llevó a cabo la demostración a este problema en la Universidad de Lausana, Suiza y que fue coronada, lo que se interpreta que fue galardonada.

Díaz (2005) consigna la obra "Dinámica interna del globo" en su investigación sobre la evolución de las ideas astronómicas en Costa Rica, como un documento que relaciona la causa de los sismos con los procesos astronómicos. Por la época que el Ing. Luis Matamoros desarrollaba sus hipótesis sobre el origen de los sismos, estudiosos costarricenses del tema astronómico como Guillermo Molina y Pedro Nolasco Gutiérrez hacían aportes importantes en este sentido y que curiosamente Matamoros no cita en su trabajo.

Sin embargo, es Luis Matamoros quien se arriesga a escribir en nuestro país la primera investigación en Sismología que trata de explicar los sismos mediante los efectos de las corrientes telúricas que apoya en una demostración mediante un profuso desarrollo matemático. 


\section{Comentarios sobre la obra}

Luis Matamoros clasifica los sismos en dos clases: la primera que asocia con el popular término de temblores de aire, que hoy sabemos que son sismos con ondas de alta frecuencia. La explicación que da sobre estos sismos es esencialmente mecánica, pues estos obedecen a la elasticidad del planeta, pudiéndose aplicar las leyes de Newton referentes al choque de los cuerpos. El comportamiento de las partículas en la tierra las compara con el comportamiento de las masas suspendidas en el arreglo de Mariotte. Se basa en la premisa de que la masa terrestre es perfectamente elástica.

El segundo grupo de sismos los denomina eléctricos. Aquí se observa la corriente de pensamiento de Feijoo en el siglo XVIII. Matamoros tiene que resolver primero el problema de la cantidad de energía eléctrica que se necesita para producir un sismo de esta clase, y encuentra la respuesta en la interferencia de otros cuerpos celestes con nuestro planeta, proponiendo el siguiente enunciado: "La causa principal, única de estos temblores es la deformación de la masa del globo terrestre, bajo la influencia de los otros cuerpos celestes."

Esta deformación produce un elipsoide de rotación y propone que ocurre un rozamiento por frotamiento de las partículas, cuando se genera el elipsoide produce electricidad que permite que exista inducción que producirá una descarga muy superior a la de los rayos atmosféricos. Es esta descarga la que produce los sismos.

En esta hipótesis se da solución a la actividad volcánica, pues refiere que las descargas considerables de electricidad cuando atraviesan masas de agua infiltrada la descomponen en sus elementos constitutivos y se forman gran cantidad de gases combustibles que se inflaman y producen las erupciones volcánicas. De hecho, una corriente galvánica descompone el agua en sus elementos constitutivos. Aquí mezcla la hipótesis de los gases de Aristóteles y de Feijoo, aún de moda en ese tiempo. De hecho en su hipótesis, considera los volcanes como grandes acumuladores de electricidad.

Esta hipótesis, según Matamoros explica la relación entre volcanes y temblores. Sin embargo, es similar a los postulados que esgrimió Feijoo en
1756 con motivo del terremoto de Lisboa del año anterior. En esos postulados, Feijoo indica que existen masas eléctricas, las cuales, atribuye a las masas de materia inflamable. El conocimiento de la electricidad aún no estaba tan desarrollado al momento en que el ilustre benedictino lanzó su hipótesis eléctrica como causa de los sismos. Matamoros, al vivir en una época en la que se tenía mayor conocimiento sobre la electricidad, modifica sustancialmente su hipótesis, pero entonces las masas inflamables de Feijoo las asocia con los focos volcánicos que en esta hipótesis se convierten en grandes acumuladores. Además, comenta que los sismos pueden ser provocados por los gases que resultan de separar el agua en sus elementos constitutivos, amarrando de esta manera lo pensado en la corriente aristotélica.

Cita a profesores como Quet, Falb o M. Perrey, quienes tenían ideas novedosas respecto al origen de los sismos, como cuando el último se adelantó a la Tectónica de Placas, y visualizó en 1847 las hoy conocidas placas Euroasiática y Africana, pues refirió: “...cette zone semble former comme une es pece de suture encore mal établie entre l'Europe et le continente africain" (Rodríguez, 1992). Sin embargo, afirma que las ideas de esos científicos "... no llenan muchas lagunas que quedan en la ciencia á este respecto y grandes objeciones se les pueden presentar... (Matamoros, 1902). Una gran objeción que ya por 1903 se pudo hacer a la hipótesis de Matamoros, es que este considera la tierra como un cuerpo homogéneo capaz de conducir la electricidad, aunque desde el siglo XVIII se conocían las propiedades conductoras de la materia, en donde no toda materia es conductora, alguna es aislante.

Debe destacarse la idea de colocar un pararrayos invertido dentro de los volcanes de tal forma que se lograra extraer toda la electricidad térrea hacia la atmósfera. Esta idea fue planteada por el académico español radicado en Costa Rica, Juan Fernández Ferraz y que sirve de comentario al final del trabajo de Matamoros. No obstante, la idea de este tipo de aparatos no fue nueva en esa época, pues Peraldo (2003) recopiló y transcribió un artículo del periódico La Gaceta de 1855 en donde se menciona “...un artificio similar al pararrayos, pero en sentido contrario, y el establecimiento de conductores metálicos con punta de platino con el 
fin de capturar la electricidad terrestre y enviarla a la atmósfera.". De haber sido cierta la hipótesis de la electricidad como causante de los sismos, se tendría que haber enviado a la atmósfera cantidades enormes de electricidad. Hubiera sido interesante que los que propusieron esta posibilidad de deshacerse de los sismos, se hubieran preguntado qué consecuencias ambientales podrían traer el enviar la electricidad terrestre a la atmósfera. Esto es interesante, pues en la búsqueda de la verdad sobre el origen de los sismos, los científicos de los dos últimos siglos que nos preceden trataron de integrar en una visión holística interesante las posibles causas, vieron a la naturaleza como un todo cuyas partes son autoajustables y automodificables, pero no tuvieron esta visión a la hora de proponer la solución al problema como lo fue el famoso pararrayos invertido.

\section{EL TRATAMIENTO FÍSICO DEL PROBLEMA}

Con el fin de sustentar su tesis filosófica sobre el origen de los sismos, Matamoros realiza una serie de razonamientos físicos mediante un tratamiento matemático que resulta en varias relaciones que considera la síntesis de su pensamiento. Resalta al inicio de su escrito la clasificación que ofrece sobre los temblores en dos clases, verticales y horizontales ondulatorios, lo que denota un razonable conocimiento del concepto de torsión dinámica, ya que para este último caso considera que es, “...como si fuerzas apareadas accionaran en sentido inverso...", lo que implica al menos una formación básica en dinámica de los medios elásticos, poco común para la época en que este personaje plantea el problema.

La relación que discute Matamoros entre la dilatación o contracción de la corteza terrestre y la temperatura del aire, pone de manifiesto el concepto integrador naturalista propio de esa época, concepto en el cual las condiciones y variaciones de uno de los componentes del sistema están siempre influenciando el estado y variaciones de los otros componentes. Sin embargo, hoy en día, a ese conjunto de componentes se le denomina sistema climático y la relación que propone entre la litosfera (su deformación) y la atmósfera (su temperatura) no es formalmente aceptada en función de las grandes diferencias energéticas involucradas y ante la ausencia de una cadena de procesos físicos que expliquen y acoplen estas dos componentes. Es así como la presencia de nubes estratificadas o cielo despejado y con tiempo caluroso no es físicamente indicativo de temblores, ni aún en el mínimo sentido estadístico. En relación con el uso del concepto de predicción de los "movimientos sísmicos", Matamoros trata de justificar la idea de que las condiciones atmosféricas y los sismos están físicamente vinculados; no obstante, acepta su complejidad y declara que no bastarían observaciones barométricas para su predicción, sino que los sismos son el producto de muchas más "influencias".

Siempre siguiendo con las ideas planteadas que relaciona los procesos tectónicos con los atmosféricos, no queda clara la forma en que Matamoros justifica su análisis matemático para explicar la I Clase de Temblores y solo esboza una pequeña idea sobre esto al finalizar con la afirmación de que "...no se han observado nunca efectos desastrosos en esta clase de temblores. Su acción es instantánea, no se repiten con frecuencia, y pasado el temblor, generalmente el tiempo cambia, debido sin duda al desequilibrio producido en la atmósfera por la vibración". Esta relación entre la meteorología y la sismología, como se discute anteriormente, era muy utilizada en la época.

Matamoros introduce también el concepto de conservación de la cantidad de movimiento ante choques elásticos. Sin embargo, aún cuando no lo explica de esta manera, en la aplicación del concepto, muestra algún grado de confusión en la notación utilizada y al discutir luego el choque de las masas usa el concepto "de velocidad por la reacción", lo cual lo induce a errores en sus análisis a pesar de que aplica correctamente el hecho físico de que después del choque las velocidades adquiridas por las partículas son inversamente proporcionales a las masas. En general, el desarrollo de este concepto es confuso, no solo por la notación utilizada, sino en su aplicación, pues iguala en algún momento las velocidades para choques elásticos y no elásticos, lo cual a todas luces es un grave error conceptual.

Es curioso cómo Matamoros desde el inicio de su explicación llama a los sismos de II Clase 
como los "eléctricos", pues según él obedecen a causas distintas a la de la I Clase. Un aspecto digno de destacar es que el autor presenta en este trabajo lo que él denomina una explicación propia "tal como lo hemos concebido y estudiado".

Su hipótesis de trabajo es la de que "la causa principal única de estos temblores es la deformación de la masa del globo terrestre, bajo la influencia de los otros cuerpos celestes". Al decir de Matamoros, sus ideas fueron elevadas ante la Universidad de Lausana, Suiza; sin embargo, no hace mención más allá de que "fue coronada", en aparente sentido de confirmada o aprobada. El problema propuesto es el de cuál forma tomaría una masa de fluido, con distribución de densidad espacialmente constante, sometida a fuerzas de atracción y repulsión y que gira sobre su propio eje. En relación con esta hipótesis de trabajo, llama la atención que su modelo básico de "masa fluida" está en claro contraste (por no indicar el grado de fluidez) con las condiciones más rígidas observadas del sistema tierra, al que le concede en todo caso, por efecto de su modelo, propiedades de deformación de un fluido.

En la ecuación , el autor no explica qué significa el término dp (diferencial de la presión como más adelante se infiere) y de nuevo la notación es confusa, ya que mezcla propiedades vectoriales con escalares en sus ecuaciones (por ejemplo, usa $\mathrm{X}$ para denotar fuerza de atracción entre las moléculas del fluido y dx para el diferencial en la coordenada $\mathrm{x}$ ). La ecuación 1 en el trabajo en discusión parece ser dimensionalmente correcta si las fuerzas actuantes son por unidad de masa. Sin embargo, Matamoros aplica este y otros conceptos, primero al mundo molecular y luego al mundo macroscópico de la Tierra y los planetas, los cuales evidentemente distan mucho del mundo molecular en sus dimensiones espaciales y temporales y sobre todo en sus propiedades físicas. Un claro ejemplo de esto es lo que él llama X, las fuerzas de atracción molecular, las cuales son de corto alcance para ser aplicadas al problema propuesto. Además de los problemas anteriores y de notación, el autor parte de una premisa física poco clara y confusa en la que no parametriza el mundo molecular en el espacio macroscópico al que luego aplica sus resultados. Otro aspecto importante de destacar es el hecho de que Matamoros considera a la Tierra como una "masa de fluido" en claro contraste con lo que hasta ese momento se conocía sobre su estructura externa.

Lo que Matamoros llama coeficiente newtoniano $\mathrm{K}$ (número de Gauss) parece ser el coeficiente de viscosidad de la masa de fluido. Para fluidos newtonianos este coeficiente es constante y la rigidez de deformación en el medio elástico es simplemente proporcional a la tensión o esfuerzo cortante ejercido.

También llama la atención la manera en que el autor relaciona la forma de "elipsoide de revolución" de la Tierra con las fuerzas cambiantes de los planetas (en función de su distancia y posiciones relativas en el espacio) con la deformación del elipsoide. Esta deformación, según Matamoros, produce frotamiento entre las partículas, la que a su vez, genera electricidad y calor "hasta ahora de proporciones desconocidas". La descarga de esa electricidad a diferentes profundidades es de acuerdo con Matamoros la causa de los temblores, de ahí que los denomine eléctricos. Explica las erupciones volcánicas como resultado de inflamaciones de gas combustible generadas de alguna manera por la chispa eléctrica producida y la cual descompone al agua en sus elementos.

Sin duda, el autor era para su época una persona emprendedora y de mente clara y aunque su conocimiento lo aplicaba de forma confusa e incorrecta, su esfuerzo por explicar la causa de los sismos es digno de mencionar y sus razonamientos, aunque creíbles posiblemente para la gente común, eran débilmente soportados por la física conocida de la época. Su explicación denota ingenio, el cual se percibe claramente al ofrecer la explicación de las erupciones y de los casos en que habiendo ocurrido temblores, estas no se dan. Ambos fenómenos (erupciones y temblores) tienen para el autor la misma causa, la descarga de la electricidad acumulada producida por el frotamiento molecular, en otras palabras, la combinación de ideas aristotélicas y feijonianas, como se explicó anteriormente.

Con relación a las corrientes filosóficas que utiliza para su razonamiento, Matamoros explica "la combustión o ignición" de materias más o menos inflamables al hecho de que, al producirse la chispa eléctrica y atravesar masas de agua localizadas presumiblemente en el subsuelo, esta 
descompone el agua en sus elementos "dando origen a grandes masas de gas combustible que a su vez se inflaman y produzcan las erupciones volcánicas". Esta idea es claramente contraria a la física conocida en esa época. En este particular, para Matamoros la carga eléctrica sucede únicamente en las masas volcánicas, las cuales considera sujetas de cargarse aún cuando este autor no ofrece explicación al respecto del porqué esos sitios sí se cargan de electricidad y otros no. En general, la explicación ofrecida sería que la descarga eléctrica interna conduce al temblor y este a su vez genera la erupción volcánica y no en ningún otro orden, es decir los temblores eléctricos no son producto de las erupciones.

Una vez expuesta su teoría, Matamoros intenta corroborarla con ejemplos y observaciones ocurridas desde 1888. Para ello utiliza información con las épocas de los fenómenos celestes más notables de cada año e intenta correlacionarlos subjetivamente con los temblores ocurridos. En este caso, como lo explica, la inducción eléctrica ocurre por la cercanía de otros cuerpos celestes a la Tierra. Utilizando la teoría del zig-zag que Paul Perrin expuso en 1873, en que se supone que el camino de menos resistencia al paso de una chispa tiene esa forma, Matamoros intenta explicar la relación del camino seguido por la chispa y la torsión observada en el cementerio y en las columnas cuadradas del cuartel de Alajuela después del terremoto de 1888 .

El otro argumento que discute tiene que ver con el tiempo de duración de un temblor. Considera que la teoría de gases y vapores para explicar temblores es errónea, ya que esta propone que el máximo de intensidad de un temblor debe estar al principio, cuando se produce la expansión brusca de las moléculas y luego de esto, la intensidad del sismo disminuye. Argumenta que eso no se observa, más bien algunos temblores inician con movimientos poco intensos y luego se intensifican, lo que sí se explica con su teoría de la chispa por efectos del enrarecimiento que produce la chispa al viajar. Ambas explicaciones, por supuesto, son incorrectas y denotan enormes ajustes subjetivos en la teoría para que coincida con lo observado. En la mayor parte de los casos, las observaciones sobre los sismos además no habían sido realizadas de manera nada sistemática y eran bastante subjetivas para esa época.
En relación con el efecto de los astros, Matamoros explica los huracanes, ciclones, las grandes lluvias, inundaciones y derrumbamientos, por el efecto de la conjunción de los astros y los eclipses debido a la mayor masa de atracción o inducción presente. De las explicaciones ofrecidas a los fenómenos observados, Matamoros pasa a discutir el problema del pronóstico o predicción de fenómenos basados en su teoría. Es decir, él piensa que si se conoce la línea de conjunción, seria muy probable que cerca de los puntos que correspondan a esta línea en la Tierra se pudieran producir unos u otros efectos. Si los puntos afectados estuvieran sobre el mar se producirían huracanes y tempestades, si estuvieran sobre la Tierra se generarían temblores. Matamoros va aún más allá de esta aseveración al afirmar que podría obtenerse bajo su teoría una ley basada en los efectos observados para predecir los temblores y otras perturbaciones geológicas y atmosféricas.

Matamoros demuestra interés sobre la prevención de los daños por efecto de esos fenómenos y propone algunas soluciones, que aunque parecen ingeniosas, son a todas luces físicamente imposibles. Tal es el caso del cable o "pararrayos" que al ser introducido en los volcanes transporte la electricidad a los océanos para así prevenir el exceso de carga acumulada en esos sitios y evitar las perturbaciones que de acuerdo con su teoría se generarían.

\section{COMENTARIOS FINALES}

Desde la aplicación de ideas provenientes de la filosofía clásica helenística que se mezclan con ideas provenientes de la Ilustración, Matamoros ofrece una solución que para la época y para nuestro mundo científico local pudo parecer lógica y coherente, más cuando defiende sus hipótesis con un extenso tratamiento físico-matemático. Además, muchos científicos de la época consideraban los principios en que Matamoros se basa como ciertos, en donde la relación atmósfera-espacio exterior y procesos internos del globo estaban relacionados al punto de imaginarlos como un sistema cuyos productos finales fueron las erupciones y los sismos.

En términos generales, el trabajo de Luis Matamoros refleja un intento por la correcta aplicación de los fundamentos del método científico 
utilizado en esa época. Con base en una conjugación de dos corrientes de pensamiento, una aristotélica y la otra desarrollada por Benito Feijoo, y mediante las "observaciones" que el realizó para el terremoto de Fraijanes del 30 de diciembre de 1888 y un trabajo teórico que denota algún grado de conocimiento, en especial matemático, propone una teoría para explicar el origen de los sismos. En este aspecto clasifica a los temblores en dos grupos, los verticales y los "eléctricos". A estos últimos dedica prácticamente todo el artículo discutido, en la búsqueda de una explicación física. Una vez propuesta su teoría, intenta con algunos ejemplos y casos observados darle la solidez física necesaria para ser aceptada por el mundo científico de la época. Más aún, intenta con base en una propuesta de correlación entre las posiciones de los planetas y sus movimientos relativos con respecto a la Tierra predecir, utilizando una "ley" basada en esa asociación matemática, qué tipo de fenómenos se podría producir en la Tierra dependiendo de las posiciones de los planetas y de dónde en la Tierra se proyectarían el o los puntos de inducción de esas perturbaciones. A pesar de este esfuerzo, Matamoros falla conceptualmente al desarrollar su teoría, algunos de cuyos aspectos parece ignorar en la formulación, como por ejemplo la existencia de las ecuaciones de NavierStokes, quienes desde principios del siglo XIX ya habían descrito de manera independiente el problema de la deformación y movimiento en los fluidos. Otro problema que se evidencia en este trabajo, es la falta de observaciones sistemáticas de los fenómenos sísmicos y la subestimación de las enormes energías asociadas a estos movimientos y a fenómenos atmosféricos como huracanes y tempestades.

Ignorar corrientes científicas que ya venían gestándose en los grandes centros de producción científica (Europa y Estados Unidos) nos indica que todavía para principios del siglo XX, Costa Rica no apostaba a la ciencia para su desarrollo, pese a esfuerzos individuales que no eran apoyados decididamente por el sector político en aras de oficializar de una vez por todas la ciencia en Costa Rica. Peraldo (2003) recopila comentarios de personas de finales del siglo XIX que expresan apatía ante las escasas perspectivas de oficializar la ciencia como un proceso de desarrollo del país. Al pensar que si estos científicos nacionales hubieran tenido mayor apoyo del sector político mediante centros especializados de investigación, medios de difusión del conocimiento e intercambio entre científicos nacionales y extranjeros de renombre, ¿qué no pudieron efectuar en bien de la ciencia del país estas personas que trabajaron casi con las uñas en la investigación científica nacional? Esta pregunta está abierta en la actualidad.

\section{REFERENCIAS}

DÍAZ, R., 2005: Los costarricenses no han dejado de admirar las magnificencias del cielo: La evolución de las ideas astronómicas en Costa Rica (1814 - 1910).- Diálogos, Revista electrónica de Historia, 6(1), feb - ago. 2005.

FEIJOO, B., 1760a: Cartas eruditas y curiosas. Carta 25: Al Sr. D. Joseph Díaz de Guitian, residente en la Ciudad de Cádiz, sobre el Terremoto padecido el día primero de Noviembre de 1755. [asequible electrónicamente en http://www.filosofia.org/bjf/ bjfc525.htm].

FEIJOO, B., 1760b: Cartas eruditas y curiosas. Carta 27: Al mismo Señor, continuando la materia de las dos antecedentes Cartas. [asequible electrónicamente en http:// www.filosofia.org/bjf/bjfc527.htm].

FEIJOO, B., 1760c: En respuesta de otra erudita, Histórica-Moral, que, sobre el mismo asunto de Terremotos, le escribió al Autor el Señor D. Joseph Rodríguez de Arellano, Canónigo de la Santa Iglesia de Toledo, \&c. [asequible electrónicamente en http:// www.filosofia.org/bjf/bjfc529.htm].

GARCÍA, V., 2001: El pensamiento científico sobre el origen de los sismos.- En: GARCÍA, V.; 2001: Los sismos en la historia de México, Fondo de Cultura Económica, II: 71-110. 
MATAMOROS, L., 1902: Dinámica interna del globo: Investigaciones de una nueva teoría de la causa de los temblores.- 23 págs. Tipografía Nacional, San José.

LARDÉ, J., 1960: Obras completas.- 597 págs. Departamento Editorial del Ministerio de Cultura, San Salvador, El Salvador.

LAY, T. \& WALLACE, T., 1995: Modern global seismology.- 521 pág. Academic Press, California.

PERALDO, G., 1996: Amenaza de deslizamientos, sector central del cantón de Puriscal y problemática de la ciudad de Santiago. Escuela Centroamericana de Geología, Universidad de Costa Rica [Tesis Lic.].

PERALDO, G., 2003: El pensamiento científico del siglo XIX y la evolución de la geología en Costa Rica.- En: PERALDO, G. (comp.): Ciencia y técnica en la Costa Rica del siglo XIX.- Ed. Tecnol. Costa Rica, Cartago: 37-86.

RODRÍGUEZ, F., 1992: La geografía y la historia de los sismos.- Cuadernos críticos de Geografía Humana, XVI (97), Universidad de Barcelona. http://www.ub.es/geocrit/ geo97.htm
RUIZ, Á., 2003: Educación y matemáticas en la Universidad de Santo Tomás.- En: PERALDO, G. (comp.): Ciencia y técnica en la Costa Rica del siglo XIX.- Ed. Tecnol. Costa Rica, Cartago: 87-128.

WILDERMANN, M., 1911: Nociones de física.209 págs. B. Herder, Friburgo de Brisgovia, Alemania.

\section{Documentos consultados}

Archivo Nacional de Costa Rica, Planos 15927. Archivo Nacional de Costa Rica, Planos 20860. Archivo Nacional de Costa Rica, Planos 1244. Archivo Nacional de Costa Rica, Planos 12515. Archivo Nacional de Costa Rica, Planos 839. Archivo Nacional de Costa Rica, Planos 21616. Archivo Nacional de Costa Rica, Planos 12961. Archivo Nacional de Costa Rica, Planos 203. Archivo Nacional de Costa Rica, Planos 12968. Archivo Nacional de Costa Rica, Planos 20845. Archivo Nacional de Costa Rica, Planos 12511. Archivo Nacional de Costa Rica, Planos 19988. LA TRIBUNA DEL 24 DE ENERO DE 1934, Francisco Faerrón "El fallecimiento del Ing. Luis Matamoros Sandoval". www.cfia.or.cr/resenna.htm 